\title{
Nonlinear polyharmonic problems with the parameter near resonance
}

\author{
Ruyun $\mathrm{Ma}^{1}$, xiaoxiao $\mathrm{Su}^{2}$, and dongliang $\mathrm{Yan}^{2}$ \\ ${ }^{1}$ Xidian University \\ ${ }^{2}$ Northwest Normal University
}

September 9, 2020

\begin{abstract}
This paper is concerned with sublinear perturbations of resonant linear polyharmonic problems. We establish some $\{\backslash$ it a priori\} bounds and use these together with Leray-Schauder continuation and bifurcation arguments to obtain extensions of some known results of Mawhin and Schmitt on the multiplicity of solutions of nonlinear elliptic eigenvalue problems with the parameter near resonance.
\end{abstract}

\section{Hosted file}

Ma-Su-Yan.pdf available at https://authorea.com/users/357511/articles/480025-nonlinearpolyharmonic-problems-with-the-parameter-near-resonance 